Artículo Original

\title{
Validación de un inmunoensayo tipo ELISA para la cuantificación de los niveles séricos de antígeno de superficie en pacientes con infección crónica por Virus de la Hepatitis B.
}

Cira V. Rodríguez-Pelier ', Arturo Chang-Monteagudo ", Yaíma Zúñiga-Rosales ${ }^{1}$, Bárbara Torres-Rives ${ }^{1}$, Goytibell Martínez-Tellez ${ }^{1}$, Deyanira la Rosa-Hernández ${ }^{2}$, Adonay Martínez-Perera ${ }^{1}$

${ }^{1}$ Centro Nacional de Genética Médica, ${ }^{2}$ Instituto de Hematología e Inmunología, ${ }^{3}$ Centro Nacional de Genética Médica

\section{RESUMEN}

Introducción. Durante la etapa aguda de la infección por el Virus de la Hepatitis B (VHB) y el período inicial de una infección crónica, el DNA está en forma episomal (libre o extracromosomal) y se replica en el hepatocito produciendo, entre otros, viriones infectivos, DNA polimerasa y antígeno de superficie del virus (HBsAg).

Objetivo. Validar un inmunoensayo tipo ELISA para cuantificar los niveles de HBsAg en pacientes con hepatitis B crónica.

Método. Se realizó un estudio experimental de desarrollo tecnológico. Se llevó a cabo la normalización y validación de un inmunoensayo enzimático heterogéneo de doble anticuerpo para la cuantificación de HBsAg en sueros de seres humanos. 115 muestras de pacientes con hepatitis $\mathrm{B}$ crónica con resultados de carga viral se correlacionaron con las concentraciones de HBsAg.

Resultados y discusión. El método presentó coeficientes de variación intra e interensayo de
9,8 y $13,2 \%$ respectivamente. El rango de trabajo se estimó entre 0.15 y $60 \mathrm{ng} / \mathrm{mL}$. El porcentaje de recuperación estuvo entre el 90 y $110 \%$ y el ajuste lineal de la curva estándar presentó un coeficiente de determinación superior a 0,99. La correlación alcanzada entre los niveles de DNA y la concentración de HBsAg fue de $62.5 \%$.

Conclusiones. La evaluación del ELISA para la cuantificación de HBsAg desarrollado en el laboratorio mostró que cumple los parámetros de validación para su uso clínico.

Palabras clave: VH, HBsAg, ELISA, inmunoensayo, normalización, optimización, validación.

\begin{abstract}
Validation of an ELISA for quantitation of serum levels of surface antigen in patients with chronic hepatitis $B$.

Introduction. During the acute phase of infection with Hepatitis B Virus (HBV) and the initial
\end{abstract}

Autor para correspondencia: Cira V. Rodríguez-Pelier, Centro Nacional de Genética Médica, cira@cngen.sld.cu

Recibido: el 19 de abril de 2017 Aceptado para publicación: el 03 de mayo de 2017

Copyright (C) 2017 por autores (s) y Revista Biomédica.

Este trabajo esta licenciado bajo las atribuciones de la Creative Commons (CC BY).

http://creativecommons.org/licenses/by/4.0/

Este documento está disponible en https://doi.org/10.32776/revbiomed.v28i3.549

Vol. 28, No. 3, septiembre-diciembre de 2017 


\section{Rodríguez-Pelier et al}

period of chronic infection, DNA is episomally (free or extrachromosomal) and replicates in hepatocytes producing among other virions, DNA polymerase and virus surface antigen (HBsAg).

Objective. Validate an ELISA to quantify HBsAg levels in patients with chronic hepatitis B.

Method. An experimental study of technological developmentwas conducted. The normalization and validation of heterogeneous double antibody enzyme linked inmunoassay for quantitation of HBsAg in human sera was carried out. 115 serum samples from patients with chronic hepatitis B with viral load results were correlated with its HBsAg concentrations.

Results and discussion. The developed method presented variation coefficients intra and interassay of 9.8 and $13.2 \%$ respectively. The working range was estimated between 0.15 and $60 \mathrm{ng} / \mathrm{mL}$. The recovery rate was between 90 and $110 \%$ and the linear fit of the standard curve presented a determination coefficient greater than 0,99 . The correlation between the DNA levels and HBsAg concentration was $62.5 \%$.

Conclusions. The assessment of the ELISA for quantitation of HBsAg developed in the laboratory showed that it achieved validation parameters for its clinical use.

Keywords: HBV, HBsAg, ELISA; immunoassay; standardization, optimization, validation.

\section{INTRODUCCIÓN}

La infección con el Virus de la Hepatitis B (VHB) tiene una distribución mundial que afecta a más de 240 millones de personas y causa alrededor de 1 millón de muertes anuales por fallo hepático y carcinoma hepatocelular (HCC). Las proteínas del VHB incluyen la proteína del core de la nucleocápside ( $\mathrm{HBcAg})$, la proteína de envoltura o superficie (HBsAg), y la proteína soluble de la nucleocápside (HBeAg). (1)
Diferentes marcadores subrogados que correlacionan con la clínica son usados en la práctica clínica para monitorear y predecir la progresión de la enfermedad. El HBsAg es el principal marcador de infección por $\mathrm{VHB}$, es detectable 1 a 2 semanas después de la exposición y su eliminación del torrente sanguíneo, que ocurre en muy pocos casos, se considera signo de eliminación del virus. El $\mathrm{HBeAg}$ refleja la replicación y transcripción viral activa e indica infectividad. La determinación de ADN del VHB (HBV-DNA) en el suero es otro marcador de replicación viral activa, más confiable que el HBeAg, especialmente en casos de mutantes que no son detectados por la prueba tradicional de HBsAg, en estos casos se encuentran niveles de ADN elevados de virus circulante y se relaciona con peor pronóstico. (2)

El tamizaje y confirmación de las personas infectadas con el VHB se realiza a través de la detección cualitativa del HBsAg. $(3,4)$ El HBsAg también puede brindar otras informaciones clínicas si en lugar de las tradicionales pruebas cualitativas, se emplean técnicas cuantitativas capaces de medir su concentración. $(5,6)$ Las investigaciones científicas de los últimos años han puesto en evidencia que en la Hepatitis B crónica $(\mathrm{HBc})$, los niveles de $\mathrm{HBsAg}$ se correlacionan inversamente con el control de la infección: a mayor control, menor concentración de HBsAg en suero. En el año 2008 se demostraron que en los pacientes con $\mathrm{HBc}$, los niveles en sangre de HBsAg pueden ser un marcador específico durante el monitoreo de la eficacia del tratamiento antiviral. (7) Por su parte, diferentes autores concluyeron en estudios independientes, que la cuantificación del HBsAg puede utilizarse para predecir la respuesta a diferentes medicamentos antivirales o para evaluar la efectividad de los mismos. (8-11)

Recientemente se ha propuesto el empleo de la cuantificación de HBsAg para la clasificación de los portadores inactivos, planteándose que 
Validación de un inmunoensayo tipo ELISA

junto a la determinación de los niveles de HBVDNA provee información complementaria para distinguir a los portadores de VHB de los sujetos inactivos de genotipo D. El nivel de HBsAg debe monitorearse además para detectar la seroconversión espontánea, donde sus valores disminuyen a valores inferiores a los niveles de sensibilidad de los métodos de detección. (12) La cuantificación de HBsAg combinado con la determinación de HBV-DNA puede proveer información útil en el futuro. En estudio de pacientes con $\mathrm{HBeAg}$ indetectable y genotipo D de VHB cifras de HBsAg $<1000 \mathrm{IU} / \mathrm{mL}$ y DNA $V H B \leq 2000 \mathrm{IU} / \mathrm{mL}$ puede identificar con precisión a los portadores inactivos en el 90\% de los pacientes. (2)

Se ha demostrado que existen diferencias significativas en los niveles de HBsAg entre los portadores inactivos y los pacientes con $\mathrm{HBc}$ activa y $\mathrm{HBeAg}$ negativos (CHB), revelando su uso potencial para discriminar ambos estados. Mientras los niveles de HBsAg correlacionan fuertemente con los niveles séricos de HBVDNA en la población global con $\mathrm{HBc}$, lo hacen solo débilmente en pacientes $\mathrm{HBeAg}$ negativos. Los niveles de HBsAg parece que no disminuyen en la misma proporción que los niveles de HBVDNA a medida que la enfermedad progresa. Por lo que la proporción de HBV-DNA/HBsAg parece ser significativamente mayor en pacientes con CHB que en los portadores inactivos. La combinación de los niveles de ambos marcadores es la que ha mostrado mejores posibilidades para diferenciar estas variantes clínicas. (13)

En los últimos años diversos estudios han demostrado que la reducción en los niveles séricos de HBsAg en pacientes tratados con PEG-interferón puede indicar la inducción satisfactoria de control inmunológico sobre el VHB, y consecuentemente predecir la respuesta al tratamiento. (14) Durante el tratamiento con PEG-interferón, la cuantificación de HBsAg es usada para identificar pacientes que no se benefician con la terapia, lo que permite que de manera temprana como en la semana 12 , pueda decidirse si el tratamiento debe ser cambiado o detenido. (15) Sim embargo, aunque el efecto del tratamiento con análogos nucleósidos (NAs) sobre los niveles séricos de HBsAg es indirecto y relativamente lento, el monitoreo de los niveles de HBsAg puede proveer información complementaria a la determinación de HBVDNA y puede ayudar a mejorar la predicción de la respuesta inicial al tratamiento con NAs. (14)

La cuantificación de HBsAg además complementa la determinación de HBV-DNA en la predicción de progresión de la enfermedad hepática hacia cirrosis hepática y HCC y está fuertemente asociado con la disminución del suero del HBsAg, particularmente en pacientes con carga viral baja. (16)

Existe la disponibilidad en el mercado de métodos cualitativos para la determinación del HBsAg, no así de cuantitativos. Actualmente existen sistemas para cuantificar la concentración del HBsAg que son sencillos de operar por su alto nivel de automatización. Debido a la reciente aparición de estos métodos comerciales, como "Architect QT assay" (Abbott) y "Elecsys II Quantassay" (Roche), la cuantificación del HBsAg ha vuelto a cobrar interés en la comunidad científica internacional. (17-19)

En Cuba, se aplica la cuantificación de HBsAg tanto en la investigación como en la clínica, la cual desarrollaron a finales de los años 80 del siglo pasado un inmunoensayo enzimático tipo ELISA utilizando un anticuerpo policlonal, el cual dejó de producirse en la década del 90, por lo que actualmente no está disponible en el mercado nacional. $(20,21)$

En Cuba la detección del HBsAg se realiza con medios de diagnóstico de producción nacional, pero actualmente no existe este sistema para cuantificar este marcador en sueros de seres humanos. Teniendo en cuenta las aplicaciones de la cuantificación del HBsAg en 


\section{Rodríguez-Pelier et al}

el seguimiento clínico de los pacientes con $\mathrm{HBc}$, para la identificación de verdaderos portadores inactivos, la predicción de la progresión de la enfermedad hepática y el monitoreo a la respuesta al tratamiento, así como el elevado costo de los métodos comerciales cuantitativos, nos propusimos validar un inmunoensayo tipo ELISA para cuantificar los niveles de HBsAg en pacientes con $\mathrm{HBc}$.

\section{MATERIAL Y MÉTODOS}

Se realizó un estudio experimental de desarrollo tecnológico en el que se llevó a cabo la normalización y validación de un inmunoensayo enzimático heterogéneo de doble anticuerpo para la cuantificación de HBsAg en sueros de seres humanos. Aprobada su ejecución por el consejo científico del Centro Nacional de Genética (CNGEN). Para la normalización y validación del inmunoensayo se siguieron los procedimientos que se establecen por el Centro para el Control Estatal de la Calidad de los Medicamentos (CECMED) del Ministerio de Salud Pública (MINSAP) de Cuba en las regulaciones 41 del año 2007 (22) y 40 del año 2014. (23) Además, se tuvieron en cuenta los criterios de la FDA. (24) Los valores de absorbancia se transforman a UI/ $\mathrm{mL}$ con un programa desarrollado por el Centro para el Control de Enfermedades, Atlanta, GA, ELISA for Windows. (30)

El estudio se realizó de acuerdo con la Declaración de Helsinki, que establece los principios éticos para las investigaciones médicas en seres humanos. (25) El consentimiento informado se entregó con tiempo suficiente para que las personas pudieran decidir libremente si deseaban ser parte de la investigación. El proyecto de esta investigación fue aprobado por el comité de ética del Centro Nacional de Genética Médica.

Para la determinación de HBsAg en el suero de los pacientes, se diseñó un sistema ELISA de doble anticuerpo (sándwich). Se empleó una placa de polietileno de 96 pocillos como fase sólida, la misma fue recubierta con un anticuerpo anti-
HBsAg monoclonal recombinante durante 20 minutos, a temperatura de $50^{\circ} \mathrm{C}$. Posteriormente se adicionaron las muestras y se incubó a $50^{\circ} \mathrm{C}$ durante $1 \mathrm{~h}$. A continuación, se añadió como conjugado un anticuerpo marcado con peroxidasa de rábano (HRP-Sigma). Como cromógeno de la reacción se empleó ortofenilendiamina (OPD, Sigma Aldrich) y como sustrato el Peróxido de Hidrógeno. La presencia de anticuerpos se detectó mediante la aparición de un complejo amarillo-naranja, cuya lectura se realizó a una longitud de onda de 492nm. Los valores de densidad óptica se trasformaron en unidades de concentración $(\mathrm{ng} / \mathrm{mL})$ mediante el programa "ELISA for Windows".

Como anticuerpo para el recubrimiento de la fase solida se utilizó Anticuerpo monoclonal anti-HBsAg de origen murino (mAb anti-HBsAg HEP-11IgG-2b) producido por el Centro de Ingeniería Genética y Biotecnología de Cuba (CIGB). $(26,29)$

El estándar secundario para la curva de calibración se preparó con antígeno recombinante obtenido y purificado en el CIGB, en una matriz compuesta por suero humano normal y albumina humana al 6\%. Se utilizó como estándar primario una preparación del CIGB (La Habana) calibrado frente al estándar internacional del Instituto Paul Erlich (Frankfurt, Alemania). (27, 28)

Se procedió de forma similar, aunque en este caso se utilizó suero de un paciente con alto título de HBsAg, para determinar el rango de aceptación del control positivo se realizaron 480 repeticiones de la muestra y se definió como el valor promedio $\pm 2 \mathrm{SD}$. La comprobación de la normalidad se llevó a cabo a través de la prueba de Kolmogorov Smirnov $(\alpha=0,05)$. (28)

Se sensibilizaron placas (Nunc Maxisorp Life Technologies, Roskilde, Denmark) con $100 \mu \mathrm{L}$ por pocillos de mAb anti-HBsAg HEP-1IgG-2b de origen murino, que tiene alta afinidad por la secuencia hidrofílica CKTCTT presente en la región inmunodominante "a" del HBsAg, diluido 
Validación de un inmunoensayo tipo ELISA

en amortiguador carbonato $0.05 \mathrm{M}, \mathrm{PH} 9.6$ a una concentración de $10 \mu \mathrm{g} / \mathrm{ml}$. Se incubó 20 minutos a $50^{\circ} \mathrm{C}$ en cámara húmeda. El anticuerpo no absorbido se eliminó con tres lavados automáticos utilizando como solución de lavado PBS al 0.15 M, pH 7.2 y Tween 20 al $0.05 \%$. $(27,28)$ La curva de calibración se preparó con seis diluciones doble seriadas del suero estándar en solución diluente (PBS al 0.15M, leche descremada al 4\% y Tween 20 al $0,05 \%$ ). Se realizaron un total de 14 curvas con tres réplicas de $100 \mu \mathrm{L}$ por pocillos para seleccionar el rango lineal. Las muestras se adicionaron diluidas $1 / 100$ en solución diluente y se incubaron las placas durante 1 hora a $50^{\circ} \mathrm{C}$. Terminado ese tiempo se realizaron los tres lavados correspondientes con solución de lavado y se añadieron $100 \mu \mathrm{L}$ del HEP-4 con HRPSigma, por pocillos a una dilución de 1/12,000 en solución diluente y se incubó a $50^{\circ} \mathrm{C}$ durante 1 hora, trascurrido el tiempo de incubación se realizaron los tres lavados y se añadió el sustrato (OPD, Sigma), Peróxido de Hidrógeno al 32\%, diluidos en amortiguador fosfato-Citrato $0.14 \mathrm{M}$, $\mathrm{pH}$ 5.5. El tiempo de reacción de la enzima con el sustrato fue de 30 minutos a temperatura entre 20 y $25^{\circ} \mathrm{C}$. Después de transcurrido ese tiempo la reacción se detuvo con $\mathrm{H}_{2} \mathrm{SO}_{4} 2 \mathrm{M}$ y las absorbancias se midieron en un espectrofotómetro a $492 \mathrm{~nm}$. Los valores de absorbancia se transforman a UI/mL. Se usó la función logisticlog de 4 parámetros para construir la curva de referencia. (30) La validación y determinación cuantitativa de HBsAg se realizó con el paquete de programas ELISA.

Para evaluar las características del método, se analizaron los parámetros: precisión, exactitud, linealidad, especificidad, límite de cuantificación y límite de detección según lo establecido en la regulación No. 47-2012 del Centro para el Control Estatal de la Calidad de los Medicamentos (CECMED) de Cuba. (23) La precisión intraensayo se evaluó procesando en la misma placa con 36 repeticiones de cuatros muestras de suero. La precisión interensayo se obtuvo mediante el montaje de muestras con iguales características a las descritas anteriormente, pero en cuatro placas que se procesaron en días diferentes por dos personas del mismo laboratorio. Para determinar la precisión tanto intraensayo como la precisión interensayo se calculó el CV mediante la fórmula: $\mathrm{CV}=(\mathrm{SD} /$ Concentración Promedio) x 100. El CV no debió superar el $10 \%$ en la precisión intraensayo y el $20 \%$ en la interensayo. Para evaluar la exactitud se efectuaron dos ensayos: recuperación y paralelismo. Para el ensayo de recuperación se realizaron seis veces el ensayo del estándar de concentración conocida en siete diluciones dobles seriadas. La exactitud se expresó matemáticamente en forma de porcentajes de recuperación y se calculó por la fórmula: valor de concentración obtenido/valor de concentración esperado x100. Se consideró que el método era exacto cuando los porcentajes de recuperación estuvieron entre el 90 y $110 \%$. El resultado de la prueba de recuperación se corroboró por la prueba $t$ de Student para muestras pareadas con el análisis de las medias de los valores obtenidos y esperados, no debiéndose encontrar diferencias significativas entre ambos grupos de datos $(p \geq$ 0.05). Para evaluar la especificidad se estudiaron seis muestras compuestas por el suero preparado para la curva de calibración de concentración de HBsAg conocida y muestras con nivel elevado de colesterol, triglicéridos, ictericia, muestras con diferentes condiciones clínicas y muestras de donantes de sangres, las que se analizaron mediante un análisis de recuperación. Se consideró buena la especificidad del método si el recobrado se encontraba entre 90 y $110 \%$. Para determinar la linealidad se realizó un análisis de regresión polinomial para valorar el ajuste de la curva. El coeficiente $\mathrm{R}^{2}$ debió ser mayor o igual a 0.98. También se tuvieron en cuenta los resultados del ensayo de paralelismo, para el cual se evaluaron tres diluciones de tres muestras y 
cuatro de la curva estándar, todos por triplicado. Para determinar el paralelismo se calculó el CV de las concentraciones de las muestras corregidas por el factor de dilución. Se consideraron óptimos los valores de CV inferiores al 10\%. (28) El límite de cuantificación se determinó como el menor valor de concentración que fue detectado con una correcta exactitud y precisión. Para el límite de detección se realizaron 288 repeticiones del control negativo, se evaluó la normalidad de la distribución y se le sumó al promedio de las densidades ópticas dos SD. La comparación de los resultados del ensayo desarrollado con los métodos comerciales cuantitativos disponibles en el mercado no se llevó a cabo. Por lo que además la completa validación analítica del ELISA desarrollado, se estudió la correlación de los resultados del mismo con la cuantificación de DNA viral que es el método empleado para el seguimiento clínico de los pacientes con HBc.

\section{RESULTADOS}

Durante la evaluación de la precisión, al analizar la repetitividad en una misma placa, con cuatro muestras a diferentes concentraciones, se obtuvo en todos los casos una imprecisión menor de 10 $\%$. En la prueba para evaluar la reproducibilidad en la que se exige un CV menor del $20 \%$ en todos los ensayos, se obtuvo CV menor del 5\% lo que demuestra una buena precisión del ensayo (Cuadro 1).

En el análisis de la exactitud del método, se alcanzaron valores de recuperación entre el 90 y 110\% (Cuadro 2).

\section{Cuadro 1}

Precisión intraensayo e interensayo del ELISA para la cuantificación de HBsAg en sueros humanos.

\begin{tabular}{|c|c|c|c|c|c|c|c|c|}
\hline \multirow{3}{*}{$\begin{array}{c}\text { Precisión } \\
\text { Muestras } \\
\mathrm{N}=12\end{array}$} & \multicolumn{6}{|c|}{ Intraensayo } & \multirow{2}{*}{\multicolumn{2}{|c|}{$\begin{array}{c}\text { Interensayo } \\
\text { Total }\end{array}$}} \\
\hline & \multicolumn{2}{|c|}{ Placa 1} & \multicolumn{2}{|c|}{ Placa 2} & \multicolumn{2}{|c|}{ Placa 3} & & \\
\hline & $\mathrm{X}^{*}$ & $\mathrm{CV} \dagger$ & $X$ & $\mathrm{CV}$ & $\mathrm{X}$ & $\mathrm{CV}$ & $\mathrm{X}$ & $\mathrm{CV}$ \\
\hline 1 & 14.4 & 0.293 & 12.0 & 6.33 & 13.6 & 1.73 & 13.32 & 1.241 \\
\hline 2 & 7.3 & 4.4 & 7.3 & 2.01 & 7.9 & 4.6 & 7.524 & 0.362 \\
\hline 3 & 2.7 & 11.06 & 2.5 & 0.348 & 2.3 & 2.6 & 2.491 & 0.171 \\
\hline 4 & 6.6 & 3.05 & 6.7 & 3.49 & 6.7 & 1.293 & 6.743 & 0.002 \\
\hline
\end{tabular}

* X: Valores promedios obtenidos de las concentraciones de HBsAg en ng/mL.

$\uparrow \mathrm{CV}$ : Coeficiente de Variación entre tres réplicas de cada muestra. 
Validación de un inmunoensayo tipo ELISA

Cuadro 2

Porcentajes de recuperación para el ELISA cuantitativo de HBsAg en sueros de seres humanos.

\begin{tabular}{ccc}
\hline $\begin{array}{c}\text { Valor esperado (ng/ } \\
\mathrm{ml})\end{array}$ & $\begin{array}{c}\text { Valor obtenido } \\
(\mathrm{ng} / \mathrm{ml})\end{array}$ & $\begin{array}{c}\text { Recuperación } \\
(\%)\end{array}$ \\
\hline 18.75 & 19.09 & 101.82 \\
9.38 & 9.39 & 100.19 \\
4.69 & 4.94 & 105.45 \\
2.34 & 2.33 & 99.50 \\
1.17 & 1.09 & 92.67 \\
0.59 & 0.59 & 101.29 \\
& & $\mathrm{p}=0.367374$
\end{tabular}

La curva estándar se evaluó con un ensayo de paralelismo o dilución. Los coeficientes $\mathrm{R}^{2}$ de la recta de mejor ajuste para cada una de las muestras y para la curva de calibración se encontraron por encima de 0,98 (Figura 1), límite de aceptación que se establece para este tipo de ensayo. Este resultado indica el comportamiento lineal del suero estándar y las muestras.

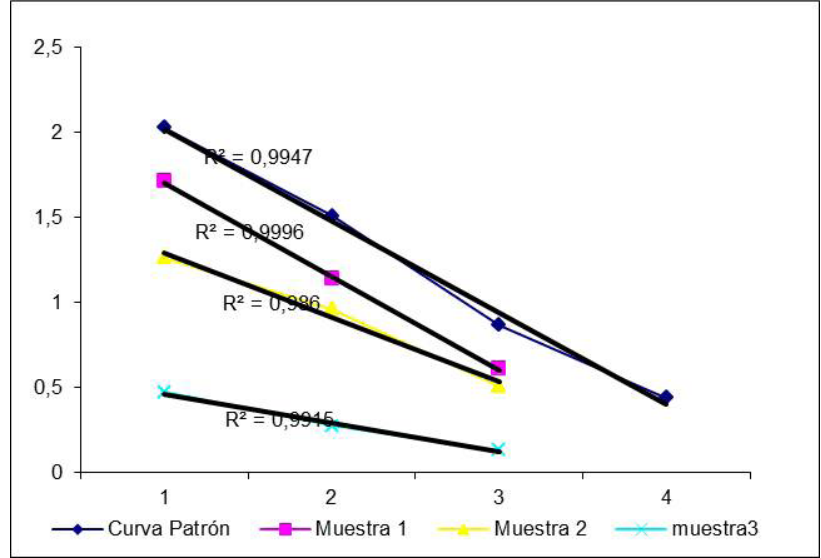

Figura 1. Ensayo de paralelismo del ELISA para cuantificar HBsAg en sueros de seres humanos.

Una vez que se corrigieron las concentraciones de las muestras multiplicando sus valores experimentales por el factor de dilución, se encontraron CV inferiores al 10\% (Cuadro 3), que no afectan su paralelismo con la curva de calibración. Se puede plantear entonces que las muestras y la curva fueron paralelas. $(23,28)$
Al realizar el estudio de la especificidad del método se comprobó que al procesar muestras donde está presente el HBsAg en un medio con diferentes contaminantes, como bilirrubina, colesterol, triglicéridos y autoanticuerpos, estos no interfirieron en los resultados, obteniéndose un porciento de recuperación en todos los casos entre 90 y 110 , lo que demuestra que el método desarrollado tiene alta especificidad para el HBsAg (Cuadro 4).

El intervalo entre el menor y el mayor nivel de detección del HBsAg que fue cuantificado con aceptable precisión y exactitud correspondió a los valores entre 0.15 y $60 \mathrm{ng} / \mathrm{mL}$. Para procesar muestras de mayor concentración sería necesario realizar diluciones de las mismas hasta que caigan en el rango de cuantificación y posteriormente, hacer la corrección multiplicando por el factor de dilución. El límite de detección fue de $0.09 \mathrm{ng} / \mathrm{mL}$.

Cuadro 3

Ensayo de paralelismo del ELISA cuantitativo de HBsAg en sueros de seres humanos.

\begin{tabular}{ccc}
\hline Muestra & CV $(\%)$ & $\mathrm{R}^{2}$ \\
\hline Curva & - & 0.99 \\
Muestra 1 (M1) & 4,8 & 0,99 \\
Muestra 2 (M2) & 3,8 & 0,98 \\
Muestra 3 (M3) & 6,1 & 0,99 \\
Muestra 4 (M4) & 5,4 & 1
\end{tabular}

Vol. 28, No. 3, septiembre-diciembre de 2017 


\section{8}

\section{Rodríguez-Pelier et al}

\section{Cuadro 4}

Resultado de la evaluación de la especificidad del método.

\begin{tabular}{cccc}
\hline Muestras & $\begin{array}{c}\text { Valor esperado } \\
\text { ng/mL }\end{array}$ & $\begin{array}{c}\text { Valor obtenido } \\
\text { ng/mL }\end{array}$ & $\begin{array}{c}\text { Porciento de } \\
\text { recuperación }\end{array}$ \\
\hline Patrón & 115 & 114.9 & 100 \\
M1* & 115 & 115.0 & 100 \\
M2† & 115 & 115.4 & 100.4 \\
M3 & 115 & 111.8 & 97.34 \\
M4§ & 115 & 111.3 & 96.87 \\
M5\| & 115 & 113.7 & 98.99 \\
M6甲 & 115 & 107.95 & 93.87
\end{tabular}

*M1: Patrón + muestra de donante de sangre.

$\uparrow$ M2: Patrón + muestra de pacientes con CBP.

†M3: Patrón + muestra de paciente con tiroiditis autoinmune y vitíligo.

§M4: Patrón + muestra con ictericia severa.

||M5: Patrón + muestra con colesterol aumentado.

qM6: Patrón + muestra con triglicéridos aumentado.

Para confirma la eficacia de la desarrollada, se analizaron un total de 125 muestras de sueros con $\mathrm{HBc}$ proporcionadas por el Instituto de Gastroenterología a nuestro laboratorio para realizar la cuantificación de la concentración de HBsAg, de las cuales 115 muestras tenían evaluación de carga viral realizadas por PCR en el Instituto de Gastroenterología, las que fueron utilizadas para realizar los análisis de correlación entre ambos marcadores. Se utilizó el factor de correlación de Pearson para evaluar la relación entre el título de HBsAg obtenido por el ELISA y los niveles de HBV-DNA determinados mediante PCR, en una escala logarítmica. Los datos se analizaron mediante la prueba de chi-cuadrada con corrección de Yates y la prueba $\mathrm{t}$ de Student cuando fue necesario.

El $100 \%$ (125 muestras) fueron antígenos positivos con un título promedio de 4,248.8 ng/ $\mathrm{mL}$ y una desviación típica de 6,775.3. De las 115 muestras con carga viral evaluadas, el $62.6 \%$ mostró correlación estadísticamente significativa entre los niveles de HBsAg y HBV-DNA (Figura 2).

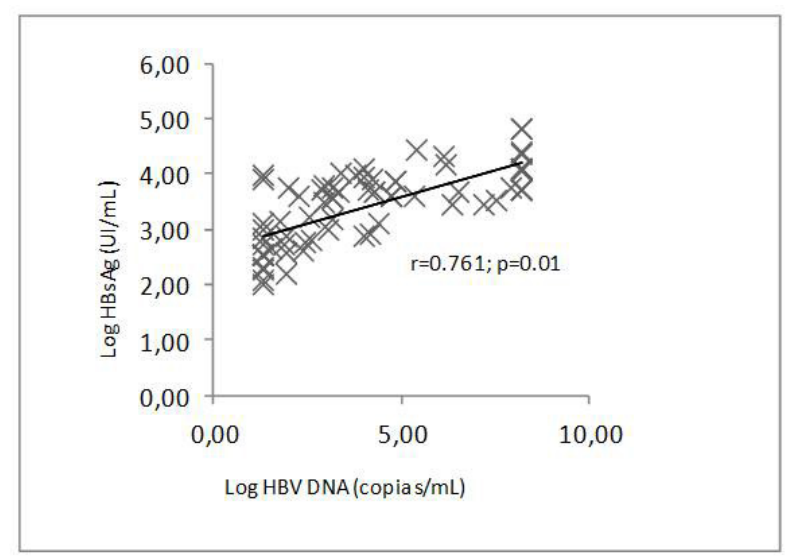

Figura 2. Correlación entre el Log de HBsAg y el Log de los niveles de ADN del VHB.

\section{DISCUSIÓN}

Al comparar la precisión del ensayo que se muestra en este estudio, con el ELISA desarrollado por Leyva y colaboradores se observa que los $\mathrm{CV}$ reportados en este último oscilaron entre el 0,77 y $3,43 \%$, lo que habla en favor de una mejor reprudicibilidad en comparación con los resultados que se obtuvieron 
Validación de un inmunoensayo tipo ELISA

en el presente estudio. Este inmunoensayo fue diseñado para evaluar el antígeno recombinante utilizado en la producción de la vacuna cubana contra la Hepatitis B (27), lo cual puede explicar la diferencia de precisión obtenida entre los dos métodos ya que en los sueros humanos existen mayor cantidad de factores que interfieren con el resultado del ensayo. (31) En cuanto a la reproducibilidad el $\mathrm{CV}$ fue mayor en nuestro ELISA fue $1.2 \%$, mientras que en el método al que hacemos referencia la reproducibilidad tuvo un $\mathrm{CV}$ máximo de $8.89 \%$ por lo que puede considerarse que el método validado por nuestro laboratorio tiene una excelente precisión interensayo. (22)

El análisis de los datos obtenidos del ensayo de recuperación, por la prueba $t$ de Student para muestras pareadas permitió considerar con un nivel de confianza del 95\% que no existieron diferencias significativas $(p=0.367374)$ entre los valores obtenidos y esperados, por lo que es posible afirmar que la cuantificación de las muestras mediante el ELISA propuesto no presentó errores sistemáticos y se corresponde con la concentración real de HBsAg en los sueros, demostrando la capacidad del método analítico para obtener resultados lo más próximos posibles al valor verdadero.

Al comparar los valores del límite de cuantificación del método desarrollado con otros métodos de cuantificación internacionales se tuvieron en cuenta los estudios de la Organización Mundial de la Salud que demuestran la no equivalencia entre las unidades de medición de las diferentes preparaciones de referencia de HBsAg. Según el Comité de Expertos para la Normalización de Biológicos, una Unidad Internacional (UI) de HBsAg es equivalente a 0.55 U "Paul Erlich Institute" (UPEI) o $0.55 \mathrm{ng} /$ mL. (32)

Teniendo en cuenta que el estándar primario que se utilizó en la presente investigación estaba calibrado en UPEI, la concentración de HBsAg en las muestras se informaron en ng (nanogramos) por $\mathrm{mL}$ (mililitros) del Sistema Internacional de Unidades.

El ELISA para evaluar HBsAg recombinante tenía un rango de trabajo entre 11.9 y 191.7 ng/mL, otro ELISA desarrollado por González Griego y colaboradores en la década de los años 80 tenía límites de trabajo entre 68 y $27 \mathrm{ng} / \mathrm{mL}$. (21) Con respecto a los métodos comerciales, "Architect QT assay" de laboratorios Abbott cuantifica entre 0.028 y $137.5 \mathrm{ng} / \mathrm{ml}$ y "Elecsys II Quantassay"de Roche, entre 0.028 y 28,600 ng/mL. (33) El “Architect QT assay" requiere de diluciones manuales de las muestras cuando estas sobrepasan su límite superior de cuantificación, pero el "Elecsys II Quantassay" tiene integrado un sistema automático de dilución que permite un amplio rango de trabajo. (34)

Del análisis del rango se deduce que la mínima concentración de muestra que pudo medirse con precisión y exactitud aceptables fue de 0.15 $\mathrm{ng} / \mathrm{mL}$. Teniendo en cuenta los resultados de investigaciones realizadas anteriormente por González Griego y colaboradores que refleja que en la distribución de la antigenemia medida por la concentración del HBsAg en portadores crónicos cubanos prevalecieron las concentraciones de HBsAg altas(10-100 $\mu \mathrm{g} / \mathrm{mL}$ ) y muy altas (mayor de $100 \mu \mathrm{g} / \mathrm{mL}$ ), que equivaldrían a $67.6 \%$ en comparación con los niveles de antigenemia medios y bajos, para el presente trabajo se fijó $a$ priori que no sería necesario que el límite inferior de cuantificación se acercara al de los ensayos de referencia. (21)

Al comparar el límite de detección del ELISA desarrollado puede afirmarse que resultó más sensible que el método de Leyva y colaboradores, el cual fue capaz de detectar hasta $1.5 \mathrm{ng} / \mathrm{mL}$. (27) Es evidente que los métodos comerciales que incluyen sustancias quimioluminiscentes como molécula señal muestran una insuperable sensibilidad analítica, no obstante, el método que se validó en la presente investigación no tiene 


\section{Rodríguez-Pelier et al}

fines de tamizaje, para lo cual ya existe un ELISA cualitativo cubano que cumple con los requisitos internacionales que se establecen para este tipo de diagnósticos. $(35,36)$

El ensayo de comparación con otro método de referencia tiene como objetivo determinar la equivalencia de métodos analíticos diferentes y permite explorar, además de la exactitud, la precisión, pero debe tenerse en cuenta que si se aprecian diferencias, estas pueden ser causadas por las propias limitaciones del método, como sería el empleo de sustratos colorimétricos en nuestra ELISA a diferencia de sustancias quimioluminiscentes de los ensayos comerciales que aumentan su sensibilidad analítica, por lo que estos estudios nunca deben emplearse de forma aislada para evaluar el error sistemático. (28) A pesar de la disponibilidad en el mercado de métodos comerciales, como "Architect QT assay" de Abbott y "Elecsys II Quantassay" de Roche, no fue posible su empleo para la comparación con los resultados de nuestro ELISA, pero se determinaron la precisión y la exactitud a través del análisis de los CV para evaluar repetitividad y reproducibilidad, y de ensayos de recuperación que permitieron el cálculo del error sistemático.

$\mathrm{Al}$ evaluar la correlación entre la cuantificación de HBsAg mediante ELISA y los niveles de HBV-DNA determinados mediante PCR hay que tener en cuenta que la disminución del DNA del VHB refleja disminución en la replicación viral, mientras que la disminución de HBsAg representa la reducción en la traducción de RNAm producido por el DNA circular covalentemente cerrado (DNAccc) transcripcionalmente activo o secuencias integradas, por tanto la cuantificación de HBsAg provee información diferente pero complementaria que permite caracterizar el estado de la infección en un individuo, (6) y la disminución o aumento del antígeno se corresponde con la disminución o aumento del DNA. La cuantificación de HBsAg en pacientes con $\mathrm{HBc}$ correlaciona con el DNAccc transcripcionalmente activo y es marcador del número de células infectadas, pero el análisis de este último solo es posible mediante técnicas complejas, restringidas a centros especializados de investigación por lo que los niveles de DNAccc no se aplican en la clínica. (6)

En el 2013 se realizó un estudio en Arabia Saudita para determinar la correlación entre la cuantificación de HBsAg y HBV-DNA en 106 pacientes, HBeAg negativos y encontraron una correlación positiva significativa entre ambos marcadores en los casos estudiados y consideran el uso de los niveles de HBsAg en la práctica clínica como un predictor de los niveles séricos de HBV-DNA. (37) En 2007 encontraron correlación entre el HBsAg y los niveles de HBV-DNA en portadores asintomáticos y en 2007 se encontró una correlación positiva entre ambos marcadores y DNAccc en pacientes con Hepatitis B HBeAg positivo. (38)

En 2014se estudiaron 167 pacientes tratados con NAs durante dos años o más, evaluando sus niveles de HBsAg y HBV-DNA, y observaron hepatocarcinogénesis en pacientes con altos niveles de HBsAg a pesar de la conversión negativa del HBV-DNA como resultado de la terapia prolongada con NAs. Por lo que consideran que para suprimir la hepatocarcinogénesis es importante controlar no solo los niveles de HBV-DNA sino además los de HBsAg en estos pacientes. (39) Recientemente se ha mostrado que pacientes $\mathrm{HBeAg}$ negativos, con HBV-DNA $<2,000 \mathrm{UI} / \mathrm{mL}$ pero con cuantificación de HBsAg $\geq 1,000 \mathrm{UI} / \mathrm{mL}$ tienen 14 veces más riesgo de padecer $\mathrm{HCC}$ que aquellos con $\mathrm{HBsAg}<1,000$ $\mathrm{UI} / \mathrm{mL}$ (40), mientras que la desaparición del HBsAg se considera que disminuye el riesgo de hepatocarcinogénesis. (41)

En 2013 demostró que los valores de HBsAg varían significativamente en las diferentes fases de la HBc y que la correlaciona con los niveles de HBV-DNA durante la inmunotolerancia, la fase positiva al HBeAg y la fase de portador inactivo, 
Validación de un inmunoensayo tipo ELISA

demuestra que la cuantificación de HBsAg puede complementar los niveles de HBV-DNA para optimizar el manejo de pacientes con $\mathrm{HBc}$ en la práctica clínica diaria. $(10,42)$

\section{CONCLUSIONES}

La determinación de la concentración de HBsAg ha sido descrita como de gran utilidad en la evaluación a lo largo del tiempo de diferentes situaciones clínicas, en función de que los niveles de dicho antígeno cambian tanto durante el curso natural de la $\mathrm{HBc}$, como durante el transcurso de la terapia. (6)

Son mucho los estudios que en los últimos años han demostrado que tanto los niveles de DNA del virus y el HBsAg disminuyen durante el curso natural de una infección crónica por VHB.

La alta precisión, exactitud y especificidad alcanzadas en la validación del ELISA desarrollado demostraron su utilidad para cuantificar el HBsAg, por tanto, en el seguimiento clínico de los pacientes con infección crónica por HBc. Los resultados obtenidos demuestran que podría ser factible el uso de las pruebas cuantitativas de HBsAg en lugar de cuantificación de HBV-DNA, como una de las estrategias utilizadas en el seguimiento regular de estos pacientes, contribuyendo a un significativo ahorro económico y también acelerar el tiempo de respuesta de las pruebas, que a su vez mejora la satisfacción del paciente.

\section{REFERENCIAS:}

1. Safaie P, Poongkunran M, Kuang PP, Javaid A, Jacobs $C$, Pohlmann R, et al. Intrahepatic distribution of hepatitis B virus antigens in patients with and without hepatocellular carcinoma. World J Gastroenterol. 2016 Mar; 22(12): 3404-11.

2. Han SH, Tran TT. Management of Chronic Hepatitis B: An Overview of Practice Guidelines for Primary Care Providers. J Am Board Fam Med. 2015 NovDec; 28(6): 6 822-37.

3. Garagulya EV, Mazurchik NV, Tarasova OI, Ogurtsov PP. HBsAg Quantitative Analysis Value for Dynamic Monitoring of Inactive Chronic Carriers of Hepatitis B Virus Infection. World J. Med. Sci. 2013 Oct-Dec; 9(4): 235-8.
4. Pai-Dhungat JV, Parikh F. Baruch Blumberg: Discoverer of Hepatitis B Virus. J Assoc Physicians India. 2015 Apr; 63(4):89.

5. Sonneveld MJ, Hansen BE, Piratvisuth T, Jia JD, Zeuzem S, Gane E, et al. Response-guided peginterferon therapy in hepatitis $\mathrm{B}$ e antigenpositive chronic hepatitis B using serum hepatitis B surface antigen levels. Hepatology. 2013 Sept; 58(3): $872-80$.

6. Liaw YF. Clinical Utility of Hepatitis B Surface Antigen Quantitation in Patients With Chronic Hepatitis B: A Review. Hepatology. 2011 Jun; 53(6): 2121-9.

7. Ozaras R, Tabak F, Tahan V, Ozturk R, Akin H, Mert $\mathrm{A}$, et al. Correlation of quantitative assay of $\mathrm{HBsAg}$ and HBV DNA levels during chronic HBV treatment. Dig Dis Sci. 2008 Nov; 53(11): 2995-8.

8. Noboru S, Kentaro M, Fuminaka S, Tsunamasa W, Shuko M, Etsuko L, et al. Application of a Newly Developed High-Sensitivity HBsAg Chemiluminescent Enzyme Immunoassay for Hepatitis B Patients with HBs. J Clin Microbiol. 2013 Nov; 51(11): 3484-91.

9. Manesis EK, Hadziyannis ES, Angelopoulou OP, Hadziyannis SJ. Prediction of treatment-related HBsAg loss in HBeAG-negative chronic hepatitis B: a clue from serum HBsAg levels. Antivir Ther. 2007 Jan; 12(1):73-82.

10. Kim YJ, Cho HC, Choi MS, Lee JH, Koh KC, Yoo $\mathrm{BC}$, et al. The change of the quantitative HBsAg level during the natural course of chronic hepatitis B. Liver Int. 2011 Jul; 31(6):817-23.

11. Vigano M, Lampertigo P. Clinical Implications of HBsAg Quantification in Patients with Chronic Hepatitis B. Saudi J Gastroenterol. 2012 Mar-Apr; 18(2): 81-6.

12. Magalhães MJ, Pedroto I. Hepatitis B Virus Inactive Carriers: Which Follow-up Strategy? GE Port J Gastroenterol. 2015 Mar-Apr; 22(2): 47-51.

13. Pita I, Horta-Vale AM, Cardoso H, Macedo G. Hepatitis B inactive carriers: An overlooked population? GE Port J Gastroenterol. 2014 Nov-Dec; 21(6): 241-9.

14. Chien-Hung C, Yi-Chun C, Sheng-Nan L, Chuan-Mo $\mathrm{L}$, Jing-Houng $\mathrm{W}$, Tsung-Hui $\mathrm{H}$, et al. Serum hepatitis $B$ surface antigen levels predict treatment response to nucleos(t)ide analogues. World J Gastroenterol. 2014 Jun; 20(24): 7686-95.

15. Martinot-Peignoux M, Lapalus $\mathrm{M}$, Asselah $\mathrm{T}$, Marcellin P. The role of HBsAg quantification for monitoring natural history and treatment outcome. Liver Int. 2013 Feb; 33 Suppl 1:125-32. 


\section{Rodríguez-Pelier et al}

16. Liu J, Lee MH, Batrla-Utermann R, Jen CL, Iloeje $\mathrm{UH}, \mathrm{Lu} \mathrm{SN}$, et al. A predictive scoring system for the seroclearance of $\mathrm{HBsAg}$ in $\mathrm{HBeAg}$-seronegative chronic hepatitis $\mathrm{B}$ patients with genotype $\mathrm{B}$ or $\mathrm{C}$ infection. J Hepatol. 2013 May; 58(5): 853-60.

17. Chen $\mathrm{CH}$, Lee CM, Wang JH, Tung HD, Hung $\mathrm{CH}, \mathrm{Lu} \mathrm{SN}$. Correlation of quantitative assay of hepatitis B surface antigen and HBV DNA levels in asymptomatic hepatitis B virus carriers. Eur J Gastroenterol Hepatol. 2004 Nov; 16(11): 1213-8.

18. Larsson SB, Eilard A, Malmstm S, Hannoun C, Dhillon AP, Norkrans G, et al. HBsAg quantification for identification oliver disease in chronic hepatitis B virus carriers. Liver Int 2014 Jul; 34(2): 38-45.

19. Wang M, Qiu N, Lu S, Xiu D, Yu J, Wang XT, et al. Serum hepatitis B surface antigen is correlated with intrahepatic total HBV DNA and cccDNA in treatment-naïve patients with chronic hepatitis B but not in patients with HBV related hepatocellular carcinoma. J Med Virol 2013 Feb; 85(2): 219-27.

20. Galban-Garcia E, Bravo-Gonzalez JR, CastanedaGuillot C, Toledo-Curbelo G, Gonzalez-Griego A, Delgado-Gonzalez MG. Field trial of the Cuban recombinant vaccine against hepatitis $B$ (Heberbiovac HB). Study in newborn infants born to AgsHB + mothers. Rev Cubana Med Trop. 1992 Jan; 44(2):149-57.

21. Santiesteban JA, Alerm-González A, GonzálezGriego A. López-Mantilla L, Marín-Padrón LC. Evaluación bioquímico-inmunológica de infectados crónicos por el virus de la hepatitis B. Comportamiento de la respuesta inmune en sus contactos familiares. Rev Cubana Invest Bioméd, 2000 Abr; 19 (1): 14-27.

22. Órgano oficial regulatorio. Centro para el Control Estatal de la Calidad de los Medicamentos (CECMED). Regulación No. 41/2007 Validación de Métodos Analíticos. Amb Reg. 2007; 00-53: 1-13.

23. Órgano oficial regulatorio. Centro para el Control Estatal de la Calidad de los Medicamentos (CECMED). Resolución No. 40/2014: Anexo 1. De las Buenas Prácticas para Laboratorio de Control de Medicamentos. Validación de Métodos Analíticos. Amb Reg. 2014; 00-215: 1-17.

24. Food and Drug Administration. FDA guidance for industry: bioanalytical method validation. Center for Drug Evaluation and Research, U.S. Department of Health and Human Services. 2001.

25. World Medical Association Declaration of Helsinki. Ethical principles for medical research involving human subjects. Clinical Review \& Education. Special Communication. 2013 Nov; 310(20): 219-4.
26. Leyva A, Franco A, Gonzalez T, Sanchez JC, Lopez I, Geada D, et al. A rapid and sensitive ELISA to quantify an HBsAg specific monoclonal antibody and a plantderived antibody during their downstream purification process. Biologicals. 2007 Mar; 35(1): 19-25.

27. Leyva A, Sanchez JC, Valdes R, Font M, Lopez L, Hernandez N, et al. A combinatorial strategy of a new monoclonal ELISA and immunoaffinity chromatography using sodium deoxycholate to increase the recovery of multimeric proteins like r-HBsAg. Talanta. 2010 Apr; 81(1-2): 314-9.

28. Ochoa RF. Técnicas inmunoenzimáticas en el desarrollo clínico de vacunas. La Habana: Finlay Ediciones; 2013. [Fecha de acceso octubre 2016] Disponible en: https://www. researchgate.net/publication/303249195_Tecnicas inmunoenzimaticas_en_el_desarrollo_clinico de_vacunas_Immunoenzymatic_assays_for_the clinical_development_of_vaccines.

29. Izquierdo M, Cosme K, Garcia G, Martinez Y, Vega M, Costa L, et al. Establecimiento de un ensayo de potencia in vivo para el antígeno de superficie recombinante del virus de la Hepatitis $B$, en vacunas monovalentes y combinadas. Vacci Monnitor. 2014 Sep-Dic; 23(3): 83-90.

30. Plikaytis BD, Carlone GM, Turner SH, Gheesling LL, Holder PF. Program ELISA user's manual. Atlanta: Centers for Disease Control and Prevention; 1993.

31. Wild D. The Immunoassay handbook. 2nd ed. London: Nature Pub. Group; 2001.

32. Ferguson $M$, Health A, Lelie N, Nubling M, Nick S, Gerlich W, et al. WHO Working Group on Hepatitis and HIV Diagnostic Kits: report of a collaborative study to 1) assess the suitability of candidate replacement international standard for $\mathrm{HBsAG}$ and a reference panel for HBsAG and 2) to calibrate the candidate standard in IU. Geneva: World Health Organization; 2003.

33. Louisirirotchanakul S, Khupulsup K, Akraekthalin S, Chan KP, Saw S, Aw TC, et al. Comparison of the technical and clinical performance of the Elecsys HBsAg II assay with the Architect, AxSym, and Advia Centaur HBsAg screening assays. J Med Virol. 2010 May; 82(5): 755-62.

34. Chan HL, Thompson A, Martinot-Peignoux M, Piratvisuth T, Cornberg M, Brunetto MR, et al. Hepatitis B surface antigen quantification: why and how to use it in 2011 - a core group report. J Hepatol. 2011 Nov; 55(5): 1121-31.

35. Huh HJ, Chae SL, Cha YJ. Comparison study with enzyme immunoassay and chemiluminescence 
Validación de un inmunoensayo tipo ELISA

immunoassay for hepatitis B virus surface antigen detection. Korean J Lab Med. 2007 Oct; 27(5): 355-9.

36. TECNOSUMA. UMELISA HBsAg Plus. [en línea] 2010. [Fecha de acceso octubre 2016]. Disponible en URL: http://www.tecnosuma.com/UM2031.htm.

37. Teriaky A, Al-Judaibi B. Correlation between HBsAg quantitation and HBV DNA in HBeAg-Negative HBV/D patients. Saudi J Gastroenterol 2013 NovDec; 19(6): 243-4

38. Chan HL, Wong VW, Tse AM, Tse CH, Chim AM, Chan HY, et al. Serum hepatitis B surface antigen quantitation can reflect hepatitis B virus in the liver and predict treatment response. Clin Gastroenterol Hepatol. 2007 Dec; 5(12): 1462-8.

39. Kawanaka M, Nishino K, Nakamura J, Oka T, Urata N, Goto D, et al. Quantitative Levels of Hepatitis B Virus DNA and Surface Antigen and the Risk of
Hepatocellular Carcinoma in Patients with Hepatitis B Receiving Long-Term Nucleos(t)ide Analogue Therapy. Liver Cancer. 2014 Mar; 3(1): 41-52.

40. Tseng TC, Liu CJ, Yang HC, Su TH, Wang CC, Chen $\mathrm{CL}$, et al. High levels of hepatitis B surface antigen increase risk of hepatocellular carcinoma in patients with low HBV load. Gastroenterol. 2012 May; 142(5): 1140-9.

41. Baran B. Nucleos(t)ide analogs in the prevention of hepatitis B virus related hepatocellular carcinoma. World J Hepatol. 2015 Jul; 7(13): 1742-54.

42. Tai-Chung T, Jia-Horng K. Clinical utility of quantitative $\mathrm{HBsAg}$ in natural history and nucleos(t) ide analogue treatment of chronic hepatitis B: new trick of old dog. J Gastroenterol. 2013 Jan; 1(48): $13-21$. 